\title{
Electron emission properties of Spindt-type platinum field emission cathodes
}

\section{$\operatorname{AUTHOR}(S):$}

Gotoh, Y; Nagao, M; Nozaki, D; Utsumi, K; Inoue, K; Nakatani, T; Sakashita, T; Betsui, K; Tsuji, H; Ishikawa, J

\section{CITATION:}

Gotoh, Y...[et al]. Electron emission properties of Spindt-type platinum field emission cathodes. JOURNAL OF APPLIED PHYSICS 2004, 95(3): 1537-1549

\section{ISSUE DATE:}

2004-02-01

URL:

http://hdl.handle.net/2433/39708

\section{RIGHT:}

Copyright 2004 American Institute of Physics. This article may be downloaded for personal use only. Any other use requires prior permission of the author and the American Institute of Physics. 


\title{
Molecular-scale noncontact atomic force microscopy contrasts in topography and energy dissipation on $c(4 \times 2)$ superlattice structures of alkanethiol self-assembled monolayers
}

\author{
Takeshi Fukuma and Takashi Ichii \\ Department of Electronic Science and Engineering, Kyoto University, Kyoto 606-8501, Japan \\ Kei Kobayashi \\ International Innovation Center, Kyoto University, Kyoto 606-8501, Japan \\ Hirofumi Yamada ${ }^{a)}$ and Kazumi Matsushige \\ Department of Electronic Science and Engineering, Kyoto University, Kyoto 606-8501, Japan
}

(Received 17 July 2003; accepted 4 November 2003)

\begin{abstract}
Alkanethiol self-assembled monolayers formed on $\mathrm{Au}(111)$ surfaces were investigated by noncontact atomic force microscopy (NC-AFM). Dodecanethiol monolayers prepared at $78^{\circ} \mathrm{C}$ were imaged by NC-AFM, which revealed that the film is composed predominantly of two different phases of $\mathrm{c}(4 \times 2)$ superlattice structures. The obtained molecular-scale NC-AFM contrasts are discussed in comparison with previously reported scanning tunneling microscopy images. We found that the energy dissipation image exhibits a clear difference in its molecular-scale contrast between the two phases. Possible origins of the difference are discussed in relation to the fluctuation and/or stability of the packing structures. (C) 2004 American Institute of Physics.
\end{abstract}

[DOI: $10.1063 / 1.1636831]$

\section{INTRODUCTION}

Alkanethiol $\quad\left[\mathrm{CH}_{3}\left(\mathrm{CH}_{2}\right)_{n-1} \mathrm{SH}, \mathrm{C}_{n}\right]$ self-assembled monolayers (SAMs) formed on $\mathrm{Au}(111)$ surfaces have attracted much attention due to their highly ordered structures and future potential applications such as for surface modifications $^{1}$ and for molecular electronic devices. ${ }^{2}$ Alkanethiol SAMs show a wide variety of structures including a number of metastable phases that molecules take during film growth ${ }^{3-6}$ and thermal desorption ${ }^{7}$ processes. However, in most cases, molecules are closely packed to form a wellordered molecular array. Molecular conformations and packing arrangements within the monolayer have been intensively studied by a variety of techniques. ${ }^{8-14}$ Studies using reflectance infrared spectroscopy revealed that the molecules take all-trans conformation with their molecular axes tilted by about $30^{\circ}$ from the surface normal. ${ }^{8-10}$ Transmission electron diffraction measurements showed that the molecules are hexagonally packed to form a $(\sqrt{3} \times \sqrt{3}) \mathrm{R} 30^{\circ}$ overlayer of the $\mathrm{Au}(111)$ lattice. ${ }^{11,12}$ Furthermore, a helium atom diffraction study revealed the existence of a larger unit cell composed of four distinct molecules corresponding to the $c(4 \times 2)$ superlattice with respect to the $(\sqrt{3} \times \sqrt{3}) \mathrm{R} 30^{\circ}$ structure. ${ }^{13}$ Although the origin of $\mathrm{c}(4 \times 2)$ unit cell has been explained by the analogy to the bulk $n$-alkane crystals ${ }^{13}$ or by the gauche defects caused by the dimerization of the molecules, ${ }^{14}$ it has been still under discussion.

Surface structures of the $c(4 \times 2)$ superlattice have been directly visualized by scanning tunneling microscopy (STM), which revealed some different packing arrangements even in a c $(4 \times 2)$ unit cell. Delamarche et al. presented molecularly

${ }^{a)}$ Electronic mail: h-yamada@kuee.kyoto-u.ac.jp resolved STM images of four different packing arrangements and referred to them as $\alpha$-, $\beta$-, $\gamma$-, and $\delta$-phases, as shown in Figs. 1(a)-1(d). ${ }^{15}$ STM contrasts they presented clearly confirmed the structural models predicted by Camillone et al. ${ }^{13}$ On the other hand, some research groups presented clear STM images revealing the existence of three different molecular contrasts in a $c(4 \times 2)$ unit cell. $^{16,17}$ The structure is schematically depicted in Fig. 1(e), and is hereafter referred to as $\epsilon$-phase. However, since STM contrast depends not only on the surface height variation but also on the electronic structure, it is still unclear whether or not the three observed contrast patterns reflect the true topography.

Noncontact atomic force microscopy (NC-AFM) using frequency modulation detection method ${ }^{18}$ enables us to achieve true atomic resolution in real space. ${ }^{19,20}$ In contrast to STM, this method can be applied to the investigation of insulating surfaces ${ }^{21,22}$ as well as on conductive ones. Since most organic materials have poor conductivity, NC-AFM applications to organic systems are of great importance. Molecularly resolved NC-AFM images of alkanethiol SAMs were first presented by Uchihashi et al. ${ }^{23}$ Subsequently, Fukuma et al. demonstrated molecular-resolution NC-AFM images of a longer-chain SAM that was hard to image by STM. ${ }^{24}$ However, detailed discussions on NC-AFM contrasts taken on $c(4 \times 2)$ superlattice structures have not been presented as yet. Two different kinds of $c(4 \times 2)$ superlattice structures have been investigated by NC-AFM in this study. Molecular-scale contrasts in the NC-AFM images are discussed in comparison with previously reported STM images.

Recently, considerable efforts have been made to understand the dissipation process of cantilever vibration energy during NC-AFM imaging. Although there still remain some important issues to be elucidated, previous studies revealed 

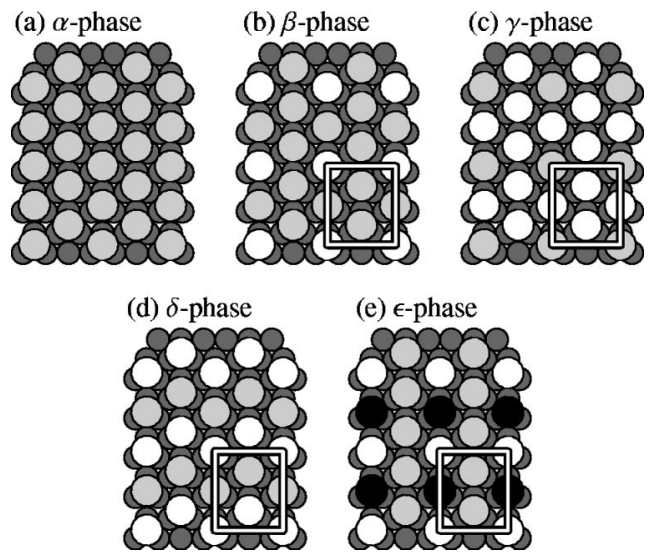

FIG. 1. Schematic models of alkanethiol SAMs on Au(111) surfaces proposed from STM images. (a) Hexagonally packed $(\sqrt{3} \times \sqrt{3})$ R3 $30^{\circ}$ structure. (b)-(e) $\mathrm{C}(4 \times 2)$ superlattice structures with a rectangular unit cell composed of four distinct molecules.

the following two important results. First, it was demonstrated that the energy dissipation can be measured on an atomic-scale resolution with an extremely high sensitivity. ${ }^{25,26}$ With a typical NC-AFM setup, energy dissipation of $0.01 \mathrm{eV}$ per cantilever oscillation cycle can be readily detected even at room temperature. ${ }^{26,27}$ Secondly, it is becoming increasingly evident that atomic-scale contrasts in dissipation images can be strongly related to the fluctuation and/or instability of tip and sample atoms. ${ }^{26,28-30}$

These two findings encouraged us to investigate energy dissipation on organic thin films by NC-AFM. As for applications to organic thin films, the influence of structural instability should be more remarkable because molecules often have loosely packed or disordered structures and the films are mostly less rigid than inorganic materials. For example, we reported that energy dissipation measured on a disordered dithiol monolayer was much larger than that on a wellordered thiol monolayer. ${ }^{31}$ Similarly, we expect that even among the well-ordered thiol monolayers, structural instability varies depending on slight differences in packing arrangements. In this study, we performed energy dissipation measurements on different kinds of $c(4 \times 2)$ superlattice structures. Molecular-scale contrasts in dissipation images are discussed in relation to the fluctuation and/or instability of the film structure.

\section{EXPERIMENTAL PROCEDURE}

The molecule used in this experiment was 1-dodecanethiol $\left[\mathrm{CH}_{3}\left(\mathrm{CH}_{2}\right)_{11} \mathrm{SH}, \mathrm{C}_{12}\right]$ purchased from Sigma-Aldrich Co., Ltd. The Au(111) surface was prepared by the evaporation of gold onto a freshly cleaved mica substrate that was heated to $420^{\circ} \mathrm{C}$. The $\mathrm{Au}(111)$ surface was immersed in $1 \mathrm{mM}$ ethanol solution of $\mathrm{C}_{12}$ for $15 \mathrm{~min}$ so that it was covered with a thiol monolayer through self-assembly. The solution was heated to $78^{\circ} \mathrm{C}$ during the dipping process to reduce defect density. ${ }^{32}$ The sample was then rinsed in pure ethanol and dried in $\mathrm{N}_{2}$ flow.

A commercially available NC-AFM apparatus (JEOL: JSPM-4500) with some modifications was used in this experiment. The original frequency shift detector was replaced with a newly developed frequency modulation detector. ${ }^{33} \mathrm{~A}$ highly doped $n$-Si cantilever (Nanosensors: $\mathrm{NCH}$ ) with a resonance frequency of about $300 \mathrm{kHz}$ and a nominal spring constant of $40 \mathrm{~N} / \mathrm{m}$ was used for NC-AFM imaging. The $Q$ factor measured under ultrahigh vacuum (UHV) conditions was about 30000 .

All the images shown in this article were taken under UHV conditions (base pressure: about $1 \times 10^{-7} \mathrm{~Pa}$ ) at room temperature. Measurements were performed in the constant frequency shift mode, where the negative shift of the cantilever resonance frequency $(\Delta f)$ induced by the tip-sample interaction was kept constant during NC-AFM imaging. The cantilever was vibrated at constant amplitude, where the vibration amplitude of the cantilever $(A)$ was kept constant by adjusting the amplitude of a cantilever excitation signal $\left(A_{\text {exc }}\right)$. In the excitation mode, energy dissipation caused by the tip-sample interaction $\left(P_{\mathrm{ts}}\right)$ can be estimated from the additional increase of $A_{\text {exc }}$ with the following equation: ${ }^{34}$

$$
P_{\mathrm{ts}}=\frac{\pi k f_{0} A^{2}}{Q_{0}}\left(\frac{A_{\mathrm{exc}}}{A_{\mathrm{exc}, 0}}-1\right),
$$

where $k$ and $f_{0}$ are spring constant and resonance frequency of a cantilever, respectively. $Q_{0}$ and $A_{\text {exc }, 0}$ are the $Q$ factor and excitation amplitude of freely oscillating cantilever, respectively. All of the dissipation values shown in this article were calculated with this equation.

\section{RESULTS AND DISCUSSION}

\section{A. Molecular-scale topographic contrasts}

Figure 2(a) is an NC-AFM image of two different $\mathrm{C}_{12}$ domains separated by a gold step. Since the monolayer was prepared at elevated temperature, the film has only some molecular-scale defects and no depressions that are usually formed by gold etching during the self-assembly process. ${ }^{32}$ Figures 2(b) and 2(c) are NC-AFM images taken on the left and right gold terraces seen in Fig. 2(a), respectively. These molecularly resolved NC-AFM images clearly show two different contrast patterns of $c(4 \times 2)$ superlattice structures. The $\mathrm{C}_{12}$ monolayer formed on the left terrace is composed of zigzag-shaped molecular rows corresponding to the $\delta$-phase model shown in Fig. 1(d), while the one formed on the right terrace has some protruded molecules forming a rectangularly shaped unit cell [ $\epsilon$-phase, Fig. 1(e)]. Higher resolution NC-AFM images of $\delta$ - and $\epsilon$-phases were obtained with larger $|\Delta f|$ values, as shown in Figs. 3(a) and 3(b), respectively. Note that these images were taken on different domains than those shown in Fig. 2, so that the orientation of the molecular rows is different. In this experiment, we could not find the other three contrast patterns of $\mathrm{c}(4 \times 2)$ superlattice structures [( $\alpha$-, $\beta$-, and $\gamma$-phases, Figs. 1(a) $-1(\mathrm{c})]$.

Although high-resolution STM images of $\mathrm{C}_{12}$ monolayers prepared with the same procedure as used in our experiment were presented by Bumm et al., ${ }^{32}$ no detailed discussions on the surface structure have been presented. The result obtained in our experiment suggested that the annealing treatment during film formation reduces not only defect density, but also the number of domains corresponding to $(\sqrt{3}$ $\times \sqrt{3})$ R3 $30^{\circ}$ structures [Fig. 1(a)]. Instead, the defect-reduced 

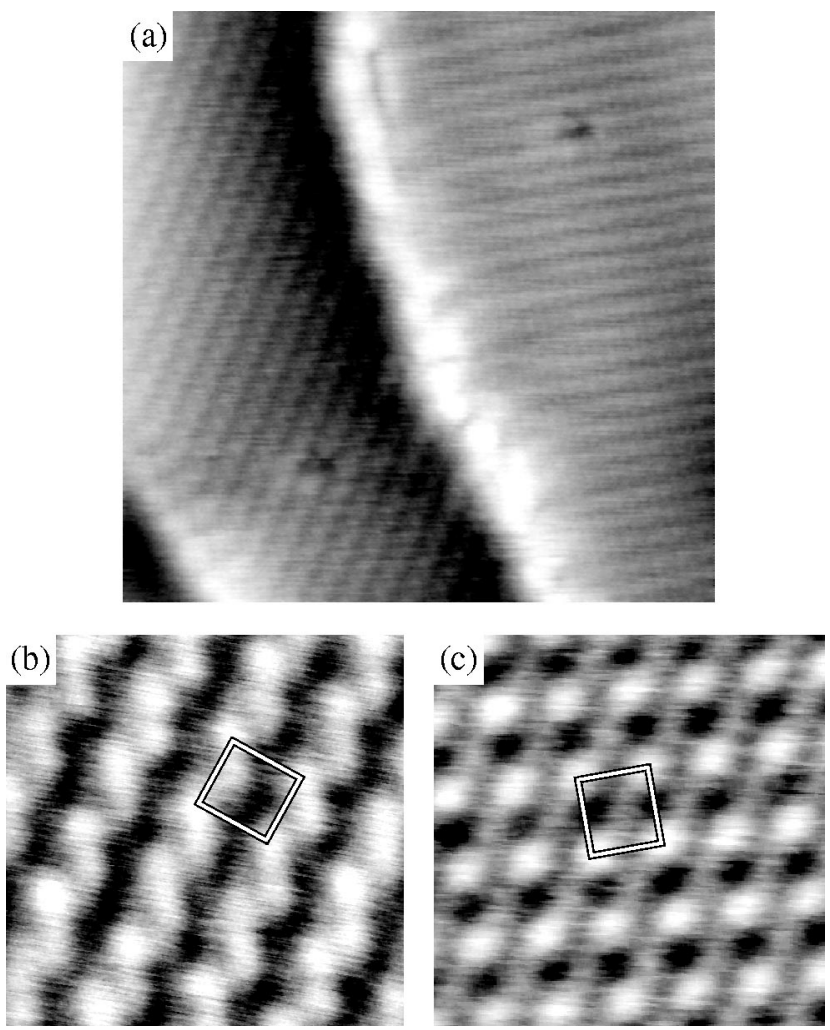

FIG. 2. NC-AFM images of $\mathrm{C}_{12}$ monolayer on $\mathrm{Au}(111)$ surface. (a) $20 \mathrm{~nm}$ $\times 20 \mathrm{~nm}, \Delta f=-70 \mathrm{~Hz}, A=5 \mathrm{~nm}$. (b) $4.5 \mathrm{~nm} \times 4.5 \mathrm{~nm}, \Delta f=-70 \mathrm{~Hz}, A$ $=5 \mathrm{~nm}$. (c) $4.5 \mathrm{~nm} \times 4.5 \mathrm{~nm}, \Delta f=-50 \mathrm{~Hz}, A=5 \mathrm{~nm}$.

SAMs are comprised predominantly of $\mathrm{c}(4 \times 2)$ superlattice structures, especially in $\delta$ - and $\epsilon$-phases. In general, an annealing treatment during film formation often brings the film structure to a thermodynamic equilibrium. The result indicated that $\delta$-and $\epsilon$-phases might be thermodynamically more stable than the other packing arrangements.

While the upper and the lower parts in Fig. 3(a) exhibit molecular-scale features of the $\delta$-phase, the middle part of the image shows a distorted contrast due to unstable imaging conditions. Namely, as the tip was brought close to the surface, the imaging conditions, such as frequency shift and the cantilever oscillation amplitude, became so unstable that high resolution was hard to achieve on $\delta$-phase domains. On the other hand, conditions during the NC-AFM imaging of
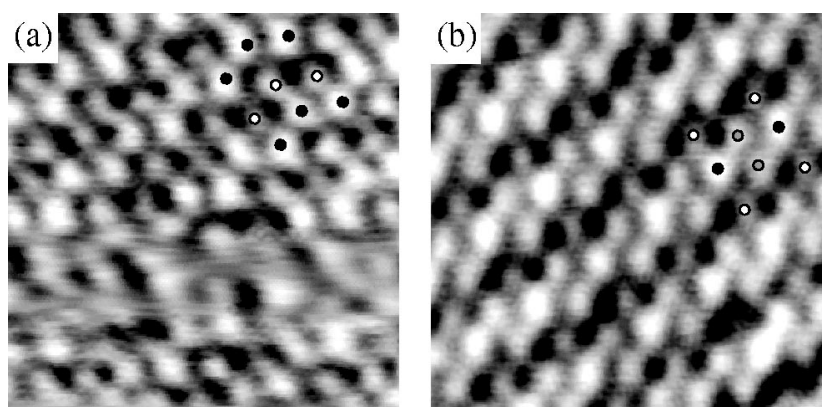

FIG. 3. NC-AFM images of $\mathrm{C}_{12}$ monolayer on $\mathrm{Au}(111)$ surface. (a) $\delta$-phase $(4.5 \mathrm{~nm} \times 4.5 \mathrm{~nm}, \Delta f=-280 \mathrm{~Hz}, A=5 \mathrm{~nm})$. (b) $\epsilon$-phase $(4.5 \mathrm{~nm} \times 4.5 \mathrm{~nm}$, $\Delta f=-260 \mathrm{~Hz}, A=5 \mathrm{~nm})$.

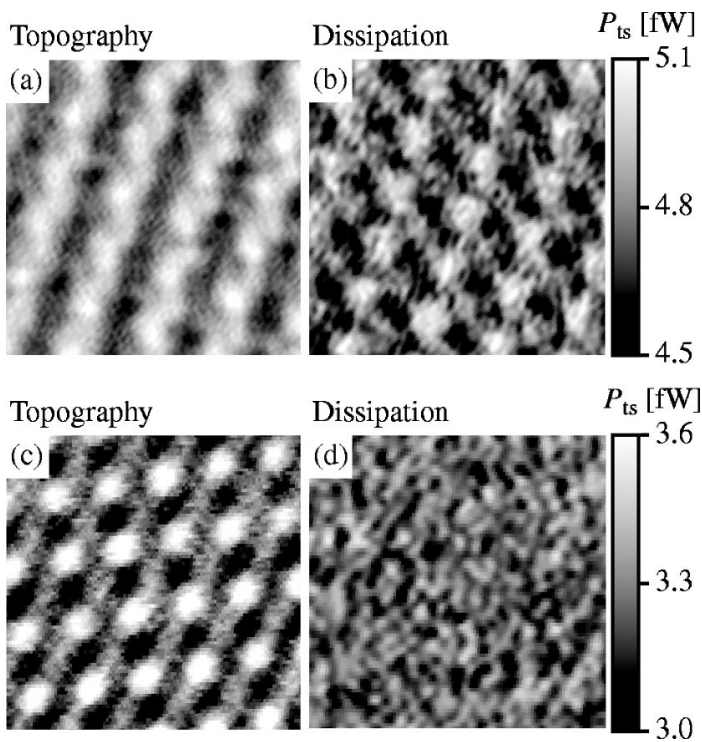

FIG. 4. NC-AFM images of $\mathrm{C}_{12}$ monolayer on $\mathrm{Au}(111)$ surface. (a) Topographic and (b) dissipation images of $\delta$-phase domain $(4.5 \mathrm{~nm}$ $\times 4.5 \mathrm{~nm}, \Delta f=-70 \mathrm{~Hz}, A=5 \mathrm{~nm}$ ). (c) Topographic and (d) dissipation images of $\epsilon$-phase domain $(4.5 \mathrm{~nm} \times 4.5 \mathrm{~nm}, \Delta f=-50 \mathrm{~Hz}, A=5 \mathrm{~nm})$.

the $\epsilon$-phase were stable enough for us to obtain highresolution NC-AFM images of $\epsilon$-phase as shown in Fig. 3(b).

In order to obtain high-resolution NC-AFM images of organic thin films, it is essential that the film structure is "rigid" enough to withstand the tip-sample interaction force. ${ }^{24}$ If the film rigidity is not high enough, molecules can be displaced by the tip-sample interaction force. Consequently, stable operation becomes difficult. In this experiment, it was found more difficult to obtain high-resolution NC-AFM images of $\delta$-phase structures than $\epsilon$-phase structures. This result suggests that the $\epsilon$-phase is more rigid and more stable against the tip-sample interaction force than the $\delta$-phase.

So far, three different molecular-scale contrast features with different brightness have been found in the $\epsilon$-phase by STM investigations. ${ }^{16,17}$ The NC-AFM image shown in Fig. 3(b) also supports the existence of three molecular-scale contrast features as indicated by the white, black and gray circles in the image. Although contrast in NC-AFM images can be affected not only by the topography itself but also by electronic structure and chemical properties of the surface, molecular-scale NC-AFM images taken on the $c(4 \times 2)$ superlattice structures are likely to represent the true surface topography. This is because the monolayer is composed of the same molecular species and the surface is terminated with chemically inert methyl end groups. Thus, the variation in the electrical and chemical properties is expected to be relatively small.

\section{B. Molecular-scale dissipation contrasts}

Figure 4 shows topographic and dissipation images taken on $\delta$ - and $\epsilon$-phases of a $\mathrm{C}_{12}$ monolayer. Dissipation images taken on the $\delta$-phase [Fig. 4(b)] clearly show molecular-scale contrasts along the zigzag-shaped molecular rows, while the one taken on the $\epsilon$-phase [Fig. 4(d)] has 
almost no contrasts, although these images were taken with the same tip and on the same sample. The observed difference in dissipation contrast was reproduced even with a different tip and on a different sample. Moreover, such a difference between the dissipation images of $\delta$ - and $\epsilon$-phases was also confirmed by dissipation imaging of decanethiol $\left(\mathrm{C}_{10}\right)$ monolayer (not shown here). Thus, the observed difference in dissipation contrast should be caused by the difference in the molecular packing arrangements.

During NC-AFM imaging, the cantilever vibration energy dissipates due to the difference between the tip-sample interaction forces in the tip approaching and retracting processes within each cycle of oscillations. In this experiment, since the energy dissipation contrast on a molecular-scale was obtained, it is difficult to explain the origin by the longrange forces such as electrostatic and van der Waals forces. Thus, it should be caused by the hysteresis in the short-range interaction forces acting directly between the tip front atom and the molecules. Furthermore, the hysteresis of the shortrange interaction force should be induced mainly by the movement of the tip front atom and the molecules interacting with it. Accordingly, the molecular-scale dissipation contrast should represent the variation in the molecular motion induced by the tip-sample interactions.

When a molecule in a monolayer is loosely bound to the surrounding molecules, it will be easily displaced by the tipsample interaction force, leading to a larger energy dissipation. Conversely, if a molecule is tightly bound by the surrounding molecules and rigid enough to withstand the tipsample interaction force, the energy dissipation measured on the molecular layer should be relatively small. In this experiment, the dissipation image taken on $\delta$-phase showed strong molecular-scale dissipation contrasts [Fig. 4(b)]. The result implies that energy dissipation is enhanced when the tip is scanned over some structurally unstable molecules in $\delta$-phase domains. On the other hand, the molecules in $\epsilon$-phase should be structurally stable since the dissipation image taken on $\epsilon$-phase showed almost no contrast [Fig. 4(d)]. In fact, the imaging conditions during the NC-AFM imaging of $\delta$-phase were more unstable than that for the $\epsilon$-phase, as shown in Fig. 3(a). This result also indicates that there were some structurally unstable molecules in the $\delta$-phase domains.

The interpretation just described is one of the possible interpretations to explain the results obtained in our experiment. Other possible contrast mechanisms cannot be completely excluded. For example, the chemical interaction potential between the tip front atom and a molecule may vary depending on a molecular position within a $c(4 \times 2)$ unit cell. Such a difference in the chemical affinity can also induce changes in the dissipation signal. ${ }^{29}$ In order to further confirm our speculation, detailed simulations with realistic tip and sample models are required.

Figure 5 shows topographic and dissipation images taken on two different $\delta$-phase domains separated by a gold step. Both images clearly show molecular-scale contrasts, revealing different orientations of the two domains. During NC-AFM imaging, the magnitude of energy dissipation was suddenly decreased near the gold step, as indicated by a

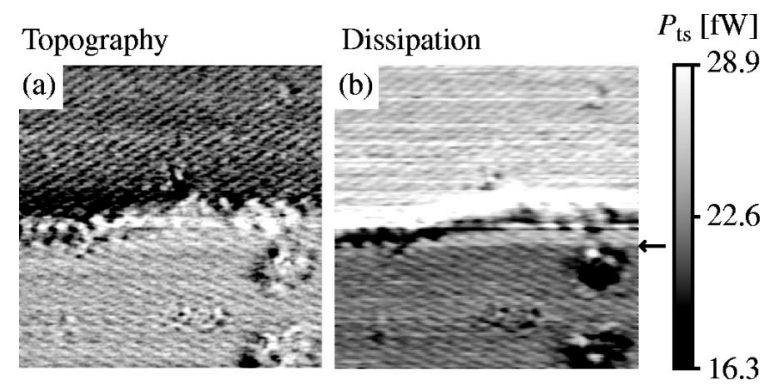

FIG. 5. NC-AFM images of $\mathrm{C}_{12}$ monolayer on $\mathrm{Au}(111)$ surface. (a) Topographic and (b) dissipation images of $\delta$-phase domain $(30 \mathrm{~nm} \times 30 \mathrm{~nm}, \Delta f$ $=-250 \mathrm{~Hz}, A=5 \mathrm{~nm}$ ).

black arrow in Fig. 5(b). Since the boundary between the bright and dark contrast features found in the dissipation image was parallel to the fast scanning direction, the decrease in energy dissipation can be assumed to be caused by some changes of the tip structure. ${ }^{30}$ However, the tip change caused no discontinuities in the molecular-scale dissipation contrast. In addition, no significant difference was found in the molecularly resolved topographic images taken before and after the tip change. These results suggest that the atomic-scale arrangement of the atoms around the tip apex was changed without a displacement of the front tip atom that contributes predominantly to the molecular-scale contrast.

In this experiment, it was found that the energy dissipation can be affected not only by surface properties, but also by the tip conditions. Thus, in order to obtain some information about the surface properties by measuring the total amount of energy dissipation, it would be required to use atomically well-defined ideal probes. However, the result also suggested that the molecular-scale dissipation contrast itself can be reproducibly obtained independently of the tip conditions. Therefore, the structural stability of the monolayers can be evaluated from the molecular-scale dissipation contrasts even when the overall dissipation is drastically changed due to an atomic-scale tip change.

\section{SUMMARY}

In this study, we investigated molecular-scale NC-AFM contrasts in topography and energy dissipation measured on $c(4 \times 2)$ superlattice structures of $C_{12}$ SAMs on Au(111) surfaces.

Molecularly resolved NC-AFM images of $\mathrm{C}_{12}$ monolayer self-assembled at $78^{\circ} \mathrm{C}$ were obtained, revealing that the monolayer is predominantly composed of $\delta$ - and $\epsilon$-phases. Moreover, three different molecular contrast features in the $\epsilon$-phase were found in the NC-AFM images similar to those previously reported for STM imaging. Results indicate that the three observed contrast features represent the true surface topography.

Dissipation images taken on the $\delta$-phase clearly show molecular-scale contrasts, while no dissipation contrasts were observed on the $\epsilon$-phase. In addition, we found it more difficult to obtain a high-resolution NC-AFM image on the $\delta$-phase than on the $\epsilon$-phase. These results suggest that there 
are some structurally unstable molecules in $\delta$-phase domain, and such a molecular-scale structural instability can be evaluated from the molecular-scale dissipation contrasts.

\section{ACKNOWLEDGMENTS}

The support of Kyoto University Venture Business Laboratory (KU-VBL), a Grant-in-Aid from the Ministry of Education, Culture, Sports, Science and Technology of Japan, and Research Fellowships of the Japan Society for the Promotion of Science for Young Scientists are gratefully acknowledged.

${ }^{1}$ J. D. Swalen et al., Langmuir 3, 932 (1987).

${ }^{2}$ Z. J. Donhauser et al., Science 292, 2303 (2001).

${ }^{3}$ G. E. Poirier and E. D. Pylant, Science 272, 1145 (1996).

${ }^{4}$ G. E. Poirier, W. P. Fitts, and J. M. White, Langmuir 17, 1176 (2001)

${ }^{5}$ W. P. Fitts, J. M. White, and G. E. Poirier, Langmuir 18, 2096 (2002).

${ }^{6}$ W. P. Fitts, J. M. White, and G. E. Poirier, Langmuir 18, 1561 (2002).

${ }^{7}$ R. Staub, M. Toerker, T. Fritz, T. Schmitz-Hübsch, F. Sellam, and K. Leo, Langmuir 14, 6693 (1998).

${ }^{8}$ M. D. Porter, T. B. Bright, D. L. Allara, and C. E. D. Chidsey, J. Am. Chem. Soc. 109, 3559 (1987).

${ }^{9}$ R. G. Nuzzo, E. M. Korenic, and L. H. Dubois, J. Chem. Phys. 93, 767 (1990).

${ }^{10}$ R. G. Nuzzo, L. H. Dubois, and D. L. Allara, J. Am. Chem. Soc. 112, 558 (1990).

${ }^{11}$ L. Strong and G. M. Whitesides, Langmuir 4, 546 (1988).

${ }^{12}$ C. E. D. Chidsey, G.-Y. Liu, G. Scoles, and J. Wang, Langmuir 6, 1804 (1990).

${ }^{13}$ N. Camillone, C. E. D. Chidsey, G.-Y. Liu, and G. Scoles, J. Chem. Phys. 98, 3503 (1993).

${ }^{14}$ P. Fenter, A. Eberhardt, and P. Eisenberger, Science 266, 1216 (1994).

${ }^{15}$ E. Delamarche, B. Michel, H. A. Biebuyck, and C. Gerber, Adv. Mater. (Weinheim, Ger.) 8, 719 (1996).
${ }^{16}$ K. Kobayashi, H. Yamada, T. Horiuchi, and K. Matsushige, Jpn. J. Appl. Phys. 37, 6183 (1998).

${ }^{17}$ J. Noh and M. Hara, Langmuir 17, 7280 (2001).

${ }^{18}$ T. R. Albrecht, P. Grütter, D. Horne, and D. Rugar, J. Appl. Phys. 69, 668 (2001).

${ }^{19}$ F. J. Giessibl, Science 267, 68 (1995).

${ }^{20}$ S. Kitamura and M. Iwatsuki, Jpn. J. Appl. Phys. 34, L1086 (1995).

${ }^{21}$ M. Bammerlin, R. Lüthi, E. Meyer, A. Baratoff, J. Lü, M. Guggisberg, C. Gerber, L. Howald, and H.-J. Güntherodt, Probe Microsc. 1, 3 (1997).

${ }^{22}$ T. Fukuma, K. Kobayashi, K. Noda, K. Ishida, T. Horiuchi, H. Yamada, and K. Matsushige, Surf. Sci. 516, 103 (2002).

${ }^{23}$ T. Uchihashi, T. Ishida, M. Komiyama, M. Ashino, Y. Sugawara, W. Mizutani, K. Yokoyama, S. Morita, H. Tokumoto, and M. Ishikawa, Appl. Surf. Sci. 157, 244 (2000).

${ }^{24}$ T. Fukuma, K. Kobayashi, T. Horiuchi, H. Yamada, and K. Matsushige, Appl. Phys. A: Mater. Sci. Process. 72, S109 (2001).

${ }^{25}$ R. Lüthi, E. Meyer, M. Bammerlin, A. Baratoff, L. Howald, C. Gerber, and H.-J. Güntherodt, Surf. Rev. Lett. 4, 1025 (1997).

${ }^{26}$ C. Loppacher, R. Bennewitz, O. Pfeiffer, M. Guggisberg, M. Bammerlin, S. Schär, V. Barwich, A. Baratoff, and E. Meyer, Phys. Rev. B 62, 13674 (2000).

${ }^{27}$ T. Fukuma, K. Umeda, K. Kobayashi, H. Yamada, and K. Matsushige, Jpn. J. Appl. Phys. 41, 4903 (2002).

${ }^{28}$ M. Gauthier and M. Tsukada, Phys. Rev. B 60, 11716 (1999).

${ }^{29}$ N. Sasaki and M. Tsukada, Jpn. J. Appl. Phys. 39, L1334 (2000).

${ }^{30}$ R. Bennewitz, A. S. Foster, L. N. Kantrovich, M. Bammerlin, C. Loppacher, S. Schär, M. Guggisberg, and E. Meyer, Phys. Rev. B 62, 2074 (2000).

${ }^{31}$ T. Ichii, T. Fukuma, K. Kobayashi, H. Yamada, and K. Matsushige, Appl. Surf. Sci. 210, 99 (2003).

${ }^{32}$ L. A. Bumm, J. J. Arnold, L. F. Charles, T. D. Dunbar, D. L. Allara, and P. S. Weiss, J. Am. Chem. Soc. 121, 8017 (1999).

${ }^{33}$ K. Kobayashi, H. Yamada, H. Itoh, T. Horiuchi, and K. Matsushige, Rev. Sci. Instrum. 72, 4383 (2001).

${ }^{34}$ B. Gotsmann, C. Seidel, B. Anczykowski, and H. Fuchs, Phys. Rev. B 60, 11051 (1999). 